\title{
Gard, Hérault
}

Vidourlenque, Vaunage et basse Vistrenque

\section{Claude Raynaud, François Favory et Laure Nuninger}

\section{(2) OpenEdition}

1 Journals

Édition électronique

URL : http://journals.openedition.org/adlfi/12170

ISSN : 2114-0502

Éditeur

Ministère de la culture

Référence électronique

Claude Raynaud, François Favory et Laure Nuninger, «Gard, Hérault », ADLFI. Archéologie de la France Informations [En ligne], Languedoc-Roussillon, mis en ligne le 01 mars 2004, consulté le 01 mai 2019. URL : http://journals.openedition.org/adlfi/12170

Ce document a été généré automatiquement le 1 mai 2019.

(c) Ministère de la Culture et de la Communication, CNRS 


\title{
Gard, Hérault
}

\author{
Vidourlenque, Vaunage et basse Vistrenque \\ Claude Raynaud, François Favory et Laure Nuninger
}

Date de l'opération : 1994 - 1999 (PI)

Inventeur(s) : Raynaud Claude (CNRS) ; Favory François (CNRS) ; Nuninger Laure (CNRS)

1 Après cinq années consacrées au programme de prospection-inventaire des cantons de Lunel et de Mauguio, sur le littoral de l'Hérault (Raynaud, Claude. 1996.) et dans la plaine du Vidourle, du Rhôny et du Vistre (Gard, 1991-1992), notre équipe a entrepris en 1994 un nouveau programme pluriannuel de prospection-inventaire portant sur la Vidourlenque, la Vaunage et la basse Vistrenque, frange méridionale de la cité antique de Nîmes (Fig. $\mathrm{n}^{\circ}$ 1 : Localisation de la zone d'étude). Ce programme a pour objectifs :

2 de compléter la campagne quinquennale consacrée aux cantons de Lunel et de Mauguio, en poursuivant l'enquête, jusqu'alors limitée à la plaine, dans les collines de la garrigue et dans la moyenne vallée du Vidourle, jusqu'à Sommières ;

3 de poursuivre la prospection de la partie orientale, au-delà du Vidourle, de la plaine de Mauguio et de Lunel, que nous avons engagée en 1991 et 1992.

On retrouve dans cette région les problèmes d'archéologie préventive liés à l'urbanisation croissante de la zone interurbaine Montpellier-Nîmes (corollaire de la densification des voies de communication et à ses effets induits - déviations, ronds-points, rocades, TGV).

\section{Cadre géographique et conditions de prospection}

5 L'originalité du projet tient à l'ampleur du terrain d'enquête. La zone retenue réunit les différents types de paysage du géosystème qui caractérise le Languedoc oriental, avec du nord au sud :

- les ultimes reliefs des garrigues calcaires sous-cévenoles, culminant entre $200 \mathrm{~m}$ et $300 \mathrm{~m}$;

- l'extrémité sud-ouest de la costière du Gard, limitée ici par un talus profondément découpé par l'érosion, et séparée des garrigues par la dépression de la Vistrenque, au pied de Nîmes ; 
- la partie orientale de la plaine de Mauguio et de Lunel, qui s'étend à la base des garrigues et de la costière jusqu'aux étangs et marais littoraux de la Petite Camargue, à l'extrémité occidentale du delta du Rhône ;

- la zone littorale, constituée ici par le milieu instable des multiples deltas qui convergeaient vers l'étang de Mauguio, derrière le cordon littoral sableux.

6 Le projet embrasse quatre régions agricoles, au sens que donnent à ce concept les agronomes, c'est-à-dire des régions dont la cohérence est définie par la similarité des caractéristiques physiques et agronomiques. Au nord-ouest, les Soubergues, coteaux dominant la vallée du Vidourle, au nord de Lunel; au nord-est, les garrigues et leurs bassins, particulièrement la Vaunage ; au centre, la plaine viticole du Bas-Languedoc, y compris le plateau-terrasse de la Costière du Gard; au sud et au sud-est, les palus de la Petite Camargue.

\section{Enjeux historiques}

7 Si de nombreux articles et monographies livrent les résultats des recherches conduites dans l'arrière-pays, ces éclairages sur certaines phases du peuplement et sur certaines formes d'habitat, essentiellement les oppida de l'âge du Fer, soulignent le contraste avec de vastes zones d'ombre: phases de peuplement peu connues comme l'Antiquité tardive et le haut Moyen Âge, secteurs géographiques non prospectés, imprécisions ou lacunes de la carte archéologique qui interdisent, actuellement, toute synthèse sur la dynamique du peuplement depuis le second âge du Fer et sur les étapes de la conquête des terres. De ce point de vue, des études restent à promouvoir sur la structuration des terroirs et l'organisation des systèmes agraires. S'imposait donc de collecter les données manquantes :

- pour caractériser le système de peuplement, trop schématiquement centré sur l'habitat perché par les premières recherches, et dont on pressentait une plus large diffusion et plus de diversité dans les formes et la hiérarchie des établissements ;

- pour analyser le processus de romanisation d'une région fortement marquée par l'occupation protohistorique, dont il convenait de caractériser l'empreinte en plaine ;

- pour individualiser les différentes phases du peuplement gallo-romain, en mesurer les rythmes singuliers en comparant les créations, les maintiens, les abandons d'habitat, en préciser les modalités de dispersion ou de regroupement de l'habitat (quid des communautés indigènes lorsqu'elles quittent leurs oppida?) et d'expansion territoriale, en essayant de proposer, comme nous l'avons fait pour le Lunellois, une carte des réseaux d'habitats aux différentes époques (Favory, François; Malvis, Jean-Michel; Mercier, Catherine ; Raynaud, Claude ; Roger, Karine. 1993. ) ;

- pour caractériser le processus de médiévalisation, à l'instar de ce que nous avons déjà tenté pour la Vaunage et la plaine et Mauguio et de Lunel [(Parodis, Anne. 1987.) ; (Parodis, Anne. 1992.)] : part de l'héritage antique, nature des habitats capables de perdurer, formes de transition, rythmes et formes de la formation villageoise, dont le paysage actuel porte encore la forte empreinte. Dans cette partie du travail, la confrontation entre les textes livrés par les cartulaires ecclésiastiques et les données de prospection est du plus grand intérêt, comme l'ont démontré les travaux d'Anne Parodi et la recherche plus récente de Catherine Mercier, qui prépare une thèse sur l'occupation de la vallée du Vidourle (université de Provence). 


\section{Premier bilan}

8 Les quatre premières années consacrées à cette entreprise ont amplement confirmé la densité de la documentation disponible, mais aussi l'urgence de sa collecte pour devancer de nombreux facteurs de destruction. Progressant à un rythme soutenu, nous avons pu étudier 2000 ha à 2500 ha par an, soit une à quatre communes, en fonction de leur taille. L'ensemble de la combe de Vaunage et des reliefs qui l'encadrent sont désormais recensés, tandis que l'étude de la vallée du Vidourle est en cours d'achèvement (couverture complète en 2000).

9 Selon les zones, c'est par deux ou par trois que l'on a pu multiplier le nombre d'établissements connus (Fig. $\mathrm{n}^{\circ} 2$ : Confrontation des données préalables à 1994 et des acquis nouveaux en Vaunage, vallée du Vistre et vallée du Vidourle (au 31 décembre 1999)). Forte de cent soixante-neuf établissements connus avant 1994 (cent trente-neuf en Vaunage, vingt pour le Vidourle, dix pour la Vistrenque), la base de données s'est enrichie avec la découverte de cinq cent huit établissements inédits. C'est donc à partir de six cent quatre-vingt-dix-neuf établissements couvrant le Néolithique final, les âges du Fer, l'époque gallo-romaine et le Moyen Âge que l'on peut revoir la dynamique du peuplement (trois cent soixante-dix-neuf en Vaunage, deux cent cinquante pour le Vidourle, soixantedix pour la Vistrenque où le travail est peu avancé à ce jour). Cette révision autorise une étude de synthèse sur le peuplement de cette région intimement liée à l'histoire de la ville de Nîmes, dont elle constitue l'un des domaines d'approvisionnement privilégié. Plus encore qu'en Lunellois où notre équipe avait mis en lumière le caractère lacunaire et l'imprécision des découvertes anciennes, la nécessité d'une prospection systématique se trouve démontrée une nouvelle fois. On en jugera en particulier à travers le graphique mettant en parallèle la situation ancienne et les découvertes récentes qui corrigent sensiblement la contribution de chaque période d'occupation et la dynamique du peuplement (Fig. ${ }^{\circ} 3$ : Dynamique du peuplement de la Vaunage, à partir de l'exemple de la commune de Calvisson (les occurrences ne correspondent pas à des établissements mais à des périodes d'occupation, qui peuvent être multiples sur un même site)). Le passage d'une documentation lacunaire à une couverture homogène ne doit pas pour autant nous abuser sur les problèmes de taphonomie liés à la conservation et à la révélation des vestiges archéologiques. Qu'il soit bien clair que nous ne prétendons pas disposer d'une documentation exhaustive ni n'affirmons avoir cerné dans son intégralité la réalité du peuplement ancien. Ne doutons pas que des travaux en profondeur réalisés en piémont des reliefs ou dans le lit des cours d'eau livreront prochainement de nouveaux établissements, imperceptibles en surface. Il s'agit plutôt de considérer que l'accroissement de la documentation permet de franchir un seuil statistique au-delà duquel des découvertes inattendues ne peuvent plus modifier de façon sensible les lignes générales fondées sur une telle base documentaire. Cette abondante collecte prend son plein intérêt au plan qualitatif, lorsque l'on considère la relative rusticité des classifications antérieures [(Pottrain, Annie. 1974.) ; (Parodis, Anne. 1987.)] qui n'avaient en particulier à leur disposition aucune grille dans la hiérarchie des établissements ni aucune mesure de l'occupation agraire, les lieux habités étant traditionnellement privilégiés par les études archéologiques. Grâce à l'enregistrement méthodique des indices hors-site, c'est désormais une étude des densités et des rythmes d'exploitation du territoire que nous pouvons mener. 


\section{En Vaunage, une densité exceptionnelle}

10

de Calvisson a été retenue comme première illustration des tendances régionales. Ce territoire de 2900 ha parait susceptible de répondre de manière significative aux questions posées par l'enquête. Alors que trente-six établissements de toutes périodes étaient connus préalablement, la prospection de tous les tènements cultivés a livré cent un sites inédits sur près de 2000 ha parcourus. L'efficacité de la prospection systématique et sa nécessité en Vaunage sont clairement établies par cette multiplication de l'information. Un seuil tout aussi appréciable est franchi au plan qualitatif grâce à l'enregistrement de tous les vestiges d'occupation y compris les indices « hors site » les plus discrets - zones d'épandage agraire ou objets prétendument isolés mais qui, par accumulation, restituent les territoires exploités autour des habitats. L'observation de coupes de terrain autorise parallèlement une première évaluation des processus d'érosion-accumulation, dimension fondamentale pour l'histoire du paysage que nous étudierons avec Guilhem Fabre (géomorphologue, CNRS, UMR 154). Enfin, la protection des sites pourra désormais être mieux assurée, plusieurs d'entre eux étant menacés à brève échéance par l'urbanisme.

On enregistre peu de nouveautés en ce qui concerne la préhistoire récente qui avait fait l'objet d'une enquête approfondie de J.-M. Roger (1986). Quelques sites mineurs dans le sud de la commune ne modifient en rien l'idée que l'on se faisait de l'occupation néolithique. Seul un petit établissement de coteau au pied d'Artillon illustre une localisation peu commune jusqu'à présent. Vient ensuite l'hiatus de l'âge du Bronze qui revêt désormais une valeur absolue en raison de la finesse du quadrillage de terrain. Peu d'originalité non plus en ce qui concerne le Bronze Final IIIB qui demeure l'apanage d'habitats perchés connus de longue date.

Au début de l'âge du Fer, le VII ${ }^{e}$ s. jusqu'alors inconnu en Vaunage, enregistre un premier établissement de faciès "suspendien ", en plaine et au bord d'un cours d'eau. Un hiatus artificiel est ainsi comblé entre le Bronze Final et le $\mathrm{VI}^{\mathrm{e}} \mathrm{s}$., qui ne livre aucun élément nouveau avant son dernier quart. On assiste alors à l'éclosion, dans la plaine de Livière, d'un réseau d'occupation tout à fait insoupçonné. Dans ce secteur se succèdent et coexistent parfois cinq groupements de cabanes. Vivace tout au long du $\mathrm{V}^{\mathrm{e}} \mathrm{s}$., cet habitat dispersé se retrouve sur quatre sites de piémont (Minteau, le Bosc). Une occupation moins dense s'observe au nord avec deux établissements, un dépôt votif ainsi qu'une aire d'épandage de fumures (Coste Basse, Ournèzes). Par sa densité - une quinzaine de sites et par l'ampleur des aménagements agraires qu'il laisse présager, l'habitat dispersé, méconnu jusqu'alors (cabanes de Congénies et de Saint-Dionisy), invite donc à repenser l'organisation du système agraire protohistorique en termes de hiérarchie des établissements, de complémentarité ou de concurrence des modes d'exploitation agricole.

La courbe des installations reflète ensuite, en négatif, l'emprise des oppida de Nages et Mauressip sur le peuplement, qui ne se disperse plus en plaine. Il faut attendre la fin du I ${ }^{\mathrm{er}} \mathrm{s}$. av. J.-C. pour enregistrer une nouvelle vague de créations qui atteint son acmé au $\mathrm{I}^{\mathrm{er}} \mathrm{s}$. de notre ère avec cinquante-cinq sites nouveaux. L'essentiel de l'occupation consiste en petites installations probablement liées aux travaux des champs ou au stockage. Une vingtaine de sites seulement accueillent des habitats, qui atteignent rarement le demihectare. Certains seulement livrent des éléments décoratifs ou thermaux pouvant trahir 
des villae, la plupart des sites moyens exprimant un niveau de vie d'une grande modestie. L'établissement le plus vaste se développe sur 2 ha en lisière du village de Calvisson où de nombreux indices invitent à identifier une agglomération. La polarisation des établissements autour de cet habitat majeur en dit long sur l'organisation du système agraire qui laisse à l'écart le grand domaine latifundiaire, que l'on trouve en lisière du territoire au pied des collines de la Liquière et d'Artillon, ou dans la vallée du Rhôny à Bizac et Girondelle.

Le III ${ }^{\mathrm{e}} \mathrm{s}$. marque un étiage, fruit d'une concentration de l'équipement agraire au sein de l'agglomération ou des établissements moyens, les petites annexes fournissant l'essentiel $\mathrm{du}$ contingent de sites désertés. Vingt-quatre sites nouveaux apparaissent durant l'Antiquité tardive, plus vastes et plus durables que les installations du Haut-Empire, essor souligné dans une étude antérieure (Parodis, Anne. 1987.). Le haut Moyen Âge marque ensuite un net recul du nombre d'établissements mais c'est peut-être aussi en négatif qu'il faut lire la courbe, comme une marque du regroupement villageois, que les textes soulignent dès le IXe $\mathrm{s}$. La prospection atteint ici ses limites, les habitats ayant peu bougé depuis lors et limitant la visibilité des vestiges. Un nouvel essor marque les $\mathrm{X}^{\mathrm{e}} \mathrm{s}$. et $\mathrm{XI}^{\mathrm{e}}$ s., avec l'apparition d'habitats dispersés, en plaine à Grésan, ou sur un coteau d'Artillon. Le XII ${ }^{e}$ s. amorce une nouvelle décrue avec la désertion des habitats dispersés, probablement sous l'effet d'une centralisation accrue des fonctions productives au village, qui affirme sa prééminence politique.

Brièvement évoqués, des résultats d'une telle richesse ne peuvent qu'inciter à poursuivre et approfondir l'étude systématique et diachronique de la Vaunage. D'ores et déjà, les menaces de destruction engendrées par l'urbanisation des abords villageois rendent urgente la mise sur pied d'une stratégie d'intervention. Plusieurs fouilles de sauvetage, menées dans la mesure du possible par notre propre équipe, sont envisageables à brève échéance et permettront d'approfondir l'acquis des prospections.

\section{Un arrière-pays : les coteaux du Vidourle}

Entre le bassin de Sommières et la plaine lunelloise, la vallée du Vidourle traverse les puechs et les petits bassins de la région des Soubergues. Ce territoire se partage entre la vallée, aux sols alluviaux déposés par le Vidourle, les faibles reliefs qui dominent d'une dizaine de mètres la vallée, et enfin quelques vallons à écoulements saisonniers. Ce paysage domine le paysage au nord-ouest du Lunellois et constitue un ensemble composite de petits reliefs de roches tendres, marnes et conglomérats calcaires, ou alluvions siliceuses rhodano-duranciennes, aisément entamés par l'érosion qui a dégagé de petits bassins, drainés par les plus modestes affluents du Vidourle. Malgré des pentes inférieures à $10 \%$, ces croupes demeurent très sensibles à l'érosion qui a souvent dénudé la roche, généralement de texture meuble. Ces caractéristiques n'offrent pas une grande richesse agrologique aux finages qui se caractérisent par les contrastes opposant les terroirs alluviaux riverains du Vidourle, les pentes érodées des puechs et les vallons colmatés de dépôts colluviaux, sols profonds mal drainés à forte endo-hydromorphie.

Le patrimoine archéologique de cette zone est longtemps resté méconnu, la carte archéologique faisant seulement état de rares découvertes anciennes, n'autorisant aucune vue d'ensemble sur le peuplement qui en première analyse paraissait clairsemé. Enfin, signalons la découverte d'une fosse de la période républicaine à la Pigneda, lors des travaux du gazoduc Artère du Midi. Cet élément isolé n'a pu être rattaché à un 
quelconque établissement, ce dernier se trouvant probablement hors d'emprise des travaux et masqué par les épais dépôts colluviaux observés à cette occasion (Garnier, Bruno ; Garnotel, Alexandrine ; Mercier, Catherine ; Raynaud, Claude. 1995.).

La prospection des communes de Boisseron, Saturargues et Saint-Sériès a confirmé cette faible emprise humaine, persistante jusqu'à une époque récente. La période néolithique a livré seulement huit établissements, tous de faible envergure, ce qui représente une installation pour plus de 220 ha, la plus faible densité observée à ce jour en Languedoc oriental. La désertion semble totale durant l'âge du Bronze et l'âge du Fer, qui n'ont livré aucun indice à ce jour. L'occupation se fait sentir à nouveau à la période gallo-romaine mais elle demeure très discrète avec une densité analogue à celle du Néolithique. Rappelons que la densité s'établit au Haut-Empire autour de trois établissements pour 100 ha en Lunellois et à neuf établissements en Vaunage. Cette faible occupation ne semble pas imputable à un problème de conservation ou de visibilité des vestiges, la vallée du Vidourle ne connaissant pas des taux d'érosion et de recouvrement sensiblement différents de ceux des zones voisines. À cette faiblesse du peuplement s'ajoutent l'exiguïté et la précarité des établissements, qui ne semblent jamais occupés plus d'une génération et correspondent plus, pour ces raisons, à des installations de colonisation agraire. Ces entreprises semblent régulièrement vouées à l'échec, avec une première vague d'établissements à la période républicaine dont aucun n'atteint le HautEmpire, une seconde phase au $\mathrm{I}^{\mathrm{er}} \mathrm{s}$. puis une troisième au $\mathrm{IV}^{\mathrm{e}} \mathrm{s}$., toutes deux aussi précaires. Il n'y a donc pas lieu de s'étonner de l'absence d'un pôle majeur de l'habitat, qui ne pouvait émerger et persister sans un réseau de mise en valeur agricole. Dans cette zone de la vallée du Vidourle, l'établissement le plus durable est celui observé en lisière du village de Saturargues, qui est occupé depuis le $\mathrm{I}^{\mathrm{er}} \mathrm{s}$. apr. J.-C. sans interruption, et semble avoir donné naissance au village médiéval : il s'agit d'un modeste établissement, probablement inférieur à 1 ha, hameau ou modeste villa, mal perçu à cause des constructions modernes. En ce qui concerne l'aménagement agraire, la prospection de surface comme les tranchées systématiques conduites lors des travaux d'installation du gazoduc Artère du Midi (travaux S. Renaud, D. Paya et A. Garnotel, 1996) aboutissent à la même observation : l'occupation reste discrète sur les collines qui dominent la vallée, où l'on observe une exploitation ténue à la période augustéenne, seulement matérialisée par deux drains et par une fosse, sur plus de $2 \mathrm{~km}$ de tracé. Cette faible emprise contraste avec la densité des aménagements agricoles dans les régions voisines.

On se gardera néanmoins de toute vision déterministe, les « contraintes » locales n'étant ni meilleures ni pires que dans les zones voisines. Si le substrat marneux ou calcaromarneux domine en effet et explique la prépondérance de sols argileux, secs une large part de l'année ou bien engorgés d'eau en saison humide, des conditions tout aussi contraignantes n'ont aucunement entravé l'exploitation du Planas de Molières, à AiguesVives, ou de certains vallons du Lunellois. On ne peut non plus incriminer les ressources en eau ni la topographie, paisible et favorable au drainage naturel. C'est probablement dans la géographie historique qu'il faut chercher des éléments d'explication, en soulignant la position intermédiaire des Soubergues entre deux zones de peuplement dynamique, autour de deux bourgades gauloises puis gallo-romaines: Sommières/ Sumidrium au nord et Ambrussum au sud. Ayant durablement fixé le peuplement et assuré leurs ressources agricoles, ces deux pôles auraient laissé subsister, sur les marges de leurs aires respectives, une périphérie longtemps délaissée. Cette polarisation de longue durée laissant persister des marges à peu près vides est un phénomène que l'on commence à 
cerner avec l'extension des prospections systématiques, notamment à la charnière entre les aires dynamiques de Mauguio et de Lattara-Sextantio. L'analyse de la morphologie agraire menée en Lunellois par François Favory met aussi l'accent sur la faible structuration antique du paysage. Contrairement à la zone littorale du Lunellois, un temps délaissée elle aussi mais devenue dynamique dès la fin de l'Antiquité, les coteaux ne seront réellement investis que très tardivement, peut-être seulement à partir de l'optimum démographique des $\mathrm{XVIII}^{\mathrm{e}} \mathrm{s}$. et XIX ${ }^{\mathrm{e}} \mathrm{s}$.

Si elle reste en deçà du nombre des établissements antiques, l'occupation médiévale se caractérise cependant par des noyaux d'occupation plus durables et plus étendus. Mais là encore, le semis se distingue nettement de ce que l'on peut observer au sud, en Lunellois, ou à l'est, en Vaunage : les habitats ne se fixent que vers la fin du haut Moyen Âge, à l'exemple du cas de Boisseron, qui connaît un premier noyau tardo-antique et altomédiéval à las Vistas, puis un second aux Tempus, dans un rayon de $500 \mathrm{~m}$ autour du castrum de Boxedone, seulement mentionné dans les textes au début du XII $\mathrm{e}$. L'habitat semble principalement localisé sur le site des Tempus, avec une église dont aucun vestige n'est connu mais dont la présence est clairement attestée par la toponymie (la Gleiza : l'Église) et les vestiges d'une nécropole. C'est donc tardivement que le peuplement s'enracine durablement, la chronologie du château et de l'habitat demeurant méconnue. L'établissement paysan a-t-il précédé et attiré la fondation castrale ? C'est ce qui semble ressortir de l'occupation des habitats de las Vistas et des Fourques dès les $\mathrm{X}^{\mathrm{e}} \mathrm{s}$. et $\mathrm{XI}^{\mathrm{e}} \mathrm{s}$., sur la hauteur et à l'écart du castrum établi en piémont, au contact de la vallée du Vidourle. Le castrum, dont la morphologie est fortement marquée par le tracé de l'enceinte, ne peut être daté qu'avec le concours des textes : castro Boxedone vers 1110 (cartulaire de Nîmes). Parmi les mentions postérieures, deux méritent attention par la relation qu'elles établissent entre le castrum et l'habitat des Tempus : de Stampucio, id est Buxedone, dans le cartulaire de Maguelone au XII ${ }^{e}$ s., et dans la même source : de Stampucio, alias de Buxedone; de même, à la fin du XIV ${ }^{\mathrm{e}} \mathrm{s}$. (pouillé), de Buxedone alias de Stempucio (Hamlin, Franck R. 1983.). Le castrum de Boisseron est évoqué et localisé en référence au site de Stampucio (L'Estampus, Lous Tempus), qui paraitt de toute évidence l'avoir précédé dans la structuration du territoire. Le castrum, simple château ou village fortifié à l'origine (?), se juxtapose à un habitat groupé antérieur. Ce dernier n'a pas résisté longtemps à l'attraction du castrum, et parait déserté au cours du XII ${ }^{\mathrm{e}} \mathrm{s}$. La minceur du mobilier recueilli lors de la prospection, rendue difficile par l'ampleur des friches et des constructions récentes, n'autorise malheureusement aucune assurance à l'égard de ces datations archéologiques.

21 C'est donc seulement vers le XII ${ }^{e} s$. que la vallée connaît un peuplement stable et durable. Dans la même perspective, rappelons la fouille de sauvetage réalisée en 1996 à SaintSériès, sur le village déserté de Saint-Félix-de-Sinistrargues, qui a mis en évidence l'aménagement de terrasses de culture au XII $\mathrm{s}$. Dans cette zone peu occupée dans l'Antiquité, il s'agit d'une mise en culture des versants, peut-être impulsée par la commanderie des Hospitaliers, à l'origine du village de Saint-Christol, autre création du Moyen Âge central (mention au XII ${ }^{e}$ s. dans le cartuaire de Maguelone).

\section{Un avant-pays : la Vistrenque}

De la garrigue aux étangs littoraux, la vallée du Vistre constitue pour la ville de Nîmes la principale voie d'accès à la Méditerranée. Dans ce couloir étroit entre costière et garrigue, 
les recherches débutent seulement et l'on ne peut faire état que de résultats partiels. Aux portes de Nîmes, les communes de Milhaud et Bernis se partagent entre la garrigue et la vallée. Les trente-quatre sites recensés précisent les grandes lignes du peuplement de la Vistrenque, entrevues à travers les découvertes anciennes et les fouilles du gazoduc Artère du Midi, en 1996. L'occupation néolithique est confirmée, principalement sur les terroirs humides des rives du Vieux Vistre. La même zone polarise un semis de petits établissements au premier âge du Fer, selon un processus de dispersion et d'extension du peuplement en plaine, déjà observé en Vaunage.

Après l'absence d'indice au second âge du Fer, le peuplement redevient sensible à la période gallo-romaine avec vingt-deux établissements. La période républicaine n'a livré que quelques indices diffus, tandis que le Haut-Empire marque une croissance nette. L'occupation change de localisation et manifeste une prédilection pour les terroirs de piémont de la garrigue, mieux drainés et plus diversifiées que ceux de la vallée. Le peuplement paraît alors dominé par quatre grands établissements, vraisemblablement deux villae sur la commune de Bernis, à quelques centaines de mètres de la via Domitia, et deux agglomérations sur la commune de Milhaud, toujours à proximité de la voie. L'occupation se densifie au Bas-Empire avec la continuité des quatre établissements majeurs, la création d'un nouveau pôle à l'ouest, et celle de petits établissements annexes. Le haut Moyen Âge demeure mal perçu malgré la découverte de trois habitats, dont l'un dans la zone palustre du Vistre qui attire à nouveau le peuplement. Les antécédents et la genèse du castrum de Bernis (mention au $\mathrm{X}^{\mathrm{e}} \mathrm{s}$.) et de la villa de Milhaud (mention au XII ${ }^{e}$ s.) demeurent mal perçus car l'extension du bâti moderne masque plusieurs centaines d'hectares autour du village, interdisant les prospections.

À l'autre extrémité de la vallée, la commune du Cailar occupe la zone de contact entre les vallées du Vidourle et du Vistre, la plaine au nord et les marais de Petite Camargue au sud. Dans cette région dominée par les terroirs alluviaux ou palustres, le peuplement néolithique demeure mal perçu, peut-être à cause des dépôts fluviaux. Un seul établissement est recensé, avec une nouvelle fois une prédilection pour les berges du Vistre. L'âge du Fer est ensuite marqué par l'émergence d'un vaste habitat en lisière de l'actuel village du Cailar. Ce site du Castellas, difficile à délimiter à cause du bâti médiéval, occupe environ 3 ha et pourrait correspondre à une bourgade protohistorique d'une certaine importance, oppidum indigène ou comptoir commercial. Au confluent du Vistre et du Rhôny, cet habitat occupait le carrefour des itinéraires reliant la mer avec les oppida de Vaunage et de Nîmes et constituait un pôle de peuplement majeur, à peu près à midistance entre les ports contemporains de Lattara et d'Espeyran. La présence d'établissements secondaires alentour et d'épandages agraires sur plusieurs kilomètres carrés marque une vaste emprise territoriale, liée à la mise en valeur de la plaine. L'occupation gallo-romaine est marquée par la dispersion de petits établissements, peutêtre organisés en réseaux autour des établissements dominants : la bourgade du Castellas, toujours occupée, et une autre agglomération potentielle à la Grande Cabane, sur la commune voisine d'Aimargues. La densité s'accroît encore au Bas-Empire avec l'extension des établissements, vastes et durables, qui formeront l'assise du maillage paroissial du haut Moyen Âge.

25 La période carolingienne est marquée par l'extension maximale du peuplement, moins en nombre d'établissements qu'en surface occupée. C'est la période la mieux connue, grâce à l'ampleur des vestiges archéologiques et aux textes du cartulaire de l'abbaye de Psalmodi, qui étend alors son emprise sur la Litoraria. Enfin, les XII ${ }^{e} s$. et XIII ${ }^{e}$ s. voient s'opérer la 
concentration dans les pôles castraux, Aimargues et le Cailar, au détriment des villae anciennes qui se trouvent majoritairement désertées avant la fin du Moyen Âge.

\section{BIBLIOGRAPHIE}

Raynaud, Claude. 1996 : «Activites du Groupe archeologique des cantons de Lunel et Mauguio en 1985-1986 », Archéologie en Languedoc, 1, p. 5-11.

Favory, FrançoisMalvis, Jean-MichelMercier, CatherineRaynaud, ClaudeRoger, Karine. 1993 : «Limitations antiques et morphologie parcellaire dans le Lunellois (Hérault) : données de fouilles récentes ", Revue archéologique de Narbonnaise, 26, p. 139-170, 23 fig.

Parodi, Anne. 1987 : « La Vaunage du $\mathrm{III}^{\mathrm{e}}$ s. au milieu du XII ${ }^{\mathrm{e}}$ s. Habitat et occupation du sol », in Archéologie du Midi médiéval, 5, p. 3-59.

Parodi, Anne. 1992 : La Plaine du Languedoc oriental au haut Moyen Âge : du IVe à la fin du XI siècle, textes et archéologie de l'espace rural, thèse de doctorat, Paris, université de Paris I, 3 vol, 860 p.

Pottrain, Annie. 1974 : L'occupation gallo-romaine de la Vaunage (Gard),, mémoire de maîtrise, université de Lille III.

Parodi, Anne. 1987 : « La Vaunage du III $^{\mathrm{e}}$ s. au milieu du XII ${ }^{\mathrm{e}}$ s. Habitat et occupation du sol », in Archéologie du Midi médiéval, 5, p. 3-59.

Parodi, Anne. 1987 : « La Vaunage du $\mathrm{III}^{\mathrm{e}}$ s. au milieu du XII ${ }^{\mathrm{e}}$ s. Habitat et occupation du sol », in Archéologie du Midi médiéval, 5, p. 3-59.

Garnier, BrunoGarnotel, AlexandrineMercier, CatherineRaynaud, Claude. 1995 : « De la ferme au village : Dassargues du V ${ }^{\mathrm{e}}$ s. au XII ${ }^{\mathrm{e}}$ s. (Lunel, Hérault) ", Archéologie du Midi médiéval, 13, p. 1-78.

Hamlin, Franck R.Cabrol, André Abbé. 1983 : Les Noms de lieux du département de l'Hérault : nouveau dictionnaire topographique et étymologique, Mèze-Montpellier, André Cabrol, 435 p.

\section{ANNEXES}


Fig. $n^{\circ} 1$ : Localisation de la zone d'étude

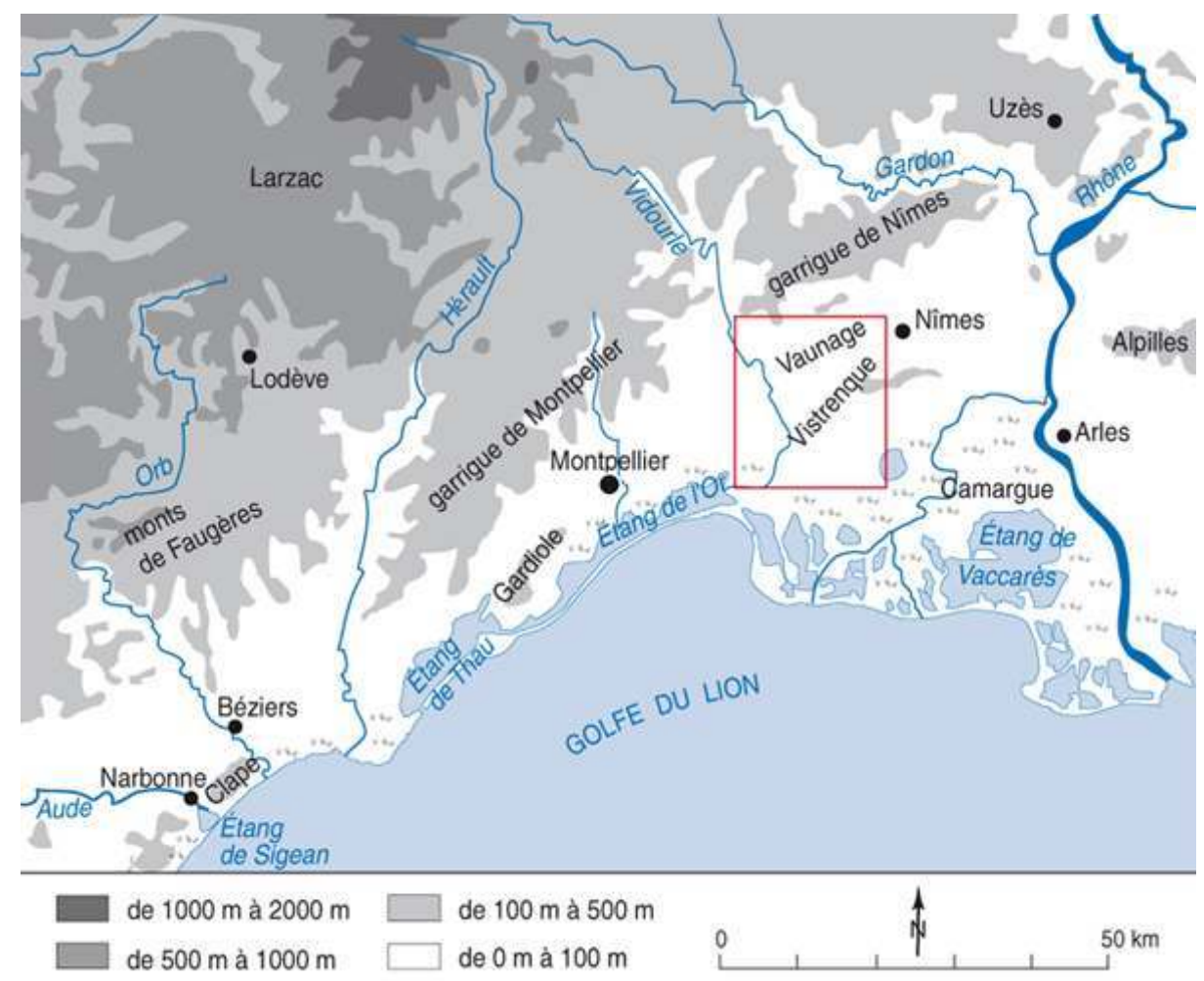

Auteur(s) : UMR 154 CNRS. Crédits : ADLFI - UMR 154 CNRS (2004) 
Fig. $n^{\circ} 2$ : Confrontation des données préalables à 1994 et des acquis nouveaux en Vaunage, vallée du Vistre et vallée du Vidourle (au 31 décembre 1999)

\begin{tabular}{|c|c|c|c|c|c|c|}
\hline \multicolumn{7}{|c|}{ VAUNAGE } \\
\hline Commune & $\begin{array}{c}\text { Superficie } \\
\left(\mathrm{km}^{2}\right)\end{array}$ & Néolithique & ấge du Fer & Gallo-romain & Moyen Áge & Total \\
\hline $\begin{array}{c}\text { Vaunage (avant } \\
1987 \text { ) }\end{array}$ & & 24 & 7 & 72 & 36 & 139 \\
\hline Boissières & 3,32 & 0 & 3 & 12 & 2 & 17 \\
\hline Calvisson & 27,94 & 7 & 16 & 67 & 10 & 100 \\
\hline Caveirac & 14,75 & 1 & 0 & 1 & 1 & 3 \\
\hline Clarensac & 14.49 & 6 & 2 & 21 & 4 & 33 \\
\hline Congénies & 8.64 & 0 & 1 & 9 & 1 & 11 \\
\hline Langlade & 8,83 & 5 & 2 & 11 & 4 & 22 \\
\hline Nages & 6.18 & 1 & 0 & 15 & 1 & 17 \\
\hline Saint-Cóme & 13,01 & 1 & 5 & 17 & 2 & 25 \\
\hline Saint-Dionisy & 3.41 & 1 & 1 & 9 & 1 & 12 \\
\hline Total & 100,57 & 46 & 37 & 234 & 62 & 379 \\
\hline \multicolumn{7}{|c|}{ VALLÉE DU VIDOURLE } \\
\hline Commune & $\begin{array}{c}\text { Superficie } \\
\left(\mathrm{km}^{2}\right)\end{array}$ & Néolithique & àge du Fer & Gallo-romain & Moyen Age & Total \\
\hline Aimargues & 26.45 & 1 & 1 & 10 & 13 & 25 \\
\hline Aubais & 11,78 & 4 & 1 & 17 & 3 & 25 \\
\hline Aujargues & 6,8 & 5 & 0 & 6 & 2 & 13 \\
\hline Boisseron & 7,36 & 6 & 0 & 5 & 2 & 13 \\
\hline Combas & 15,59 & 2 & 2 & 5 & 2 & 11 \\
\hline Fontanès & 14,44 & 8 & 6 & 24 & 7 & 45 \\
\hline Gallargues & 10,89 & 0 & 0 & 17 & 2 & 19 \\
\hline Junas & 7.45 & 4 & 2 & 4 & 4 & 14 \\
\hline Saint-Sériès & 4.51 & 1 & 0 & 5 & 1 & 7 \\
\hline Saturargues & 6 & 1 & 0 & 6 & 2 & 9 \\
\hline Sommierres & 10,36 & 3 & 1 & 13 & 2 & 19 \\
\hline Souvignargues & 11,09 & 8 & 2 & 26 & 8 & 44 \\
\hline Villevieille & 8.28 & 3 & 1 & 14 & 3 & 21 \\
\hline Total & 141 & 46 & 16 & 152 & 51 & 265 \\
\hline \multicolumn{7}{|c|}{ VALLLÉE DU VISTRE } \\
\hline Commune & $\begin{array}{c}\text { Superficie } \\
\left(\mathrm{km}^{2}\right)\end{array}$ & Néolithique & àge du Fer & Gallo-romain & Moyen Age & Total \\
\hline Bernis & 12.8 & 3 & 3 & 7 & 4 & 17 \\
\hline Le Callar & 30.01 & 7 & 2 & 20 & 7 & 36 \\
\hline Milhaud & 17,4 & 1 & 4 & 10 & 2 & 17 \\
\hline Codognan (en 2000) & & & & & & 0 \\
\hline Vergèze (en 2000) & & & & & & 0 \\
\hline Vestric (en 2000) & & & & & & 0 \\
\hline Vauvert (en 2000) & & & & & & 0 \\
\hline Total & 60.21 & 8 & 6 & 30 & 9 & 70 \\
\hline
\end{tabular}

Auteur(s) : Raynaud, Claude ; Favory, François ; Nuninger, Laure. Crédits : ADLFI - Raynaud, Claude ; Favory, François ; Nuninger, Laure (2004) 
Fig. $n^{\circ} 3$ : Dynamique du peuplement de la Vaunage, à partir de l'exemple de la commune de Calvisson (les occurrences ne correspondent pas à des établissements mais à des périodes d'occupation, qui peuvent être multiples sur un même site)

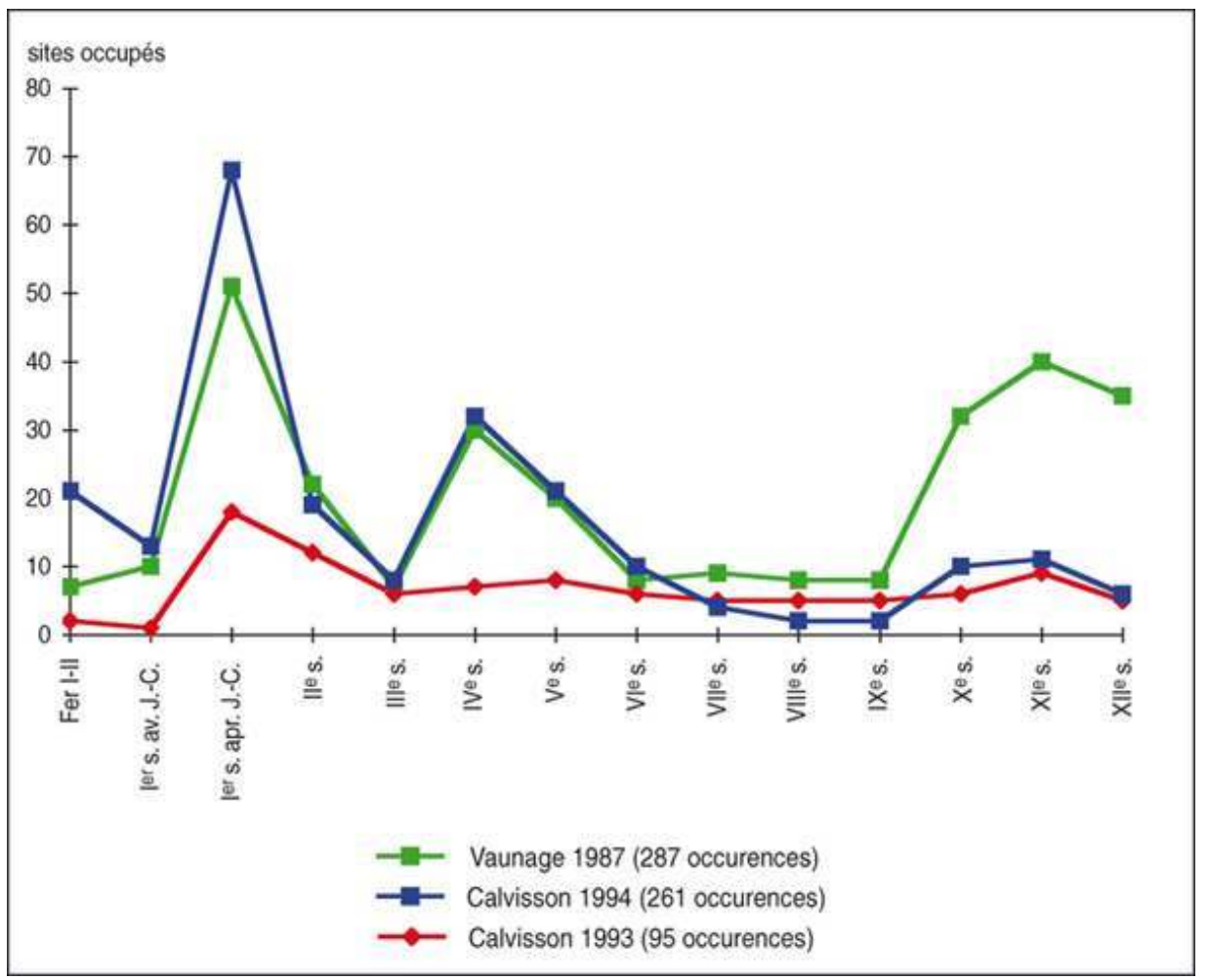

Auteur(s) : Raynaud, Claude ; Favory, François ; Nuninger, Laure. Crédits : ADLFI - Raynaud, Claude ; Favory, François; Nuninger, Laure (2004)

\section{INDEX}

Index chronologique : Antiquité romaine, Protohistoire, Paléolithique, Néolithique, Temps Modernes, ép. contemporaine, Moyen Âge operation Prospection inventaire (PI)

Index géographique : Languedoc-Roussillon, Gard (30), Hérault (34), Aimargues (30006), Milhaud, Lunel, Mauguio, Nîmes, Sommières peuple Gallo-romains

\section{AUTEURS}

\section{CLAUDE RAYNAUD}

CNRS

FRANÇOIS FAVORY

CNRS

LAURE NUNINGER

CNRS 